\title{
A Comparison of the Effect of Diurnal Temperature Range and Apparent Temperature on Cardiovascular Disease among Farmers in Qingyang, Northwest China
}

\author{
Guangyu Zhai \\ Lanzhou University of Technology \\ Jintao Qi ( $\sim 318738436 @ q q . c o m$ ) \\ Lanzhou University of Technology https://orcid.org/0000-0003-4243-4467 \\ Xuemei Zhang \\ Lanzhou University of Technology \\ Wenjuan Zhou \\ Gansu Provincial Hospital \\ Jiancheng Wang \\ Gansu Provincial Hospital
}

\section{Research}

Keywords: Diurnal temperature range, Apparent temperature, Cardiovascular diseases, Distributed lag nonlinear model, Famer

Posted Date: August 13th, 2021

DOI: https://doi.org/10.21203/rs.3.rs-770864/v1

License: (c) (1) This work is licensed under a Creative Commons Attribution 4.0 International License.

Read Full License 


\section{Abstract \\ Background}

Apparent temperature (AT) and diurnal temperature range (DTR) have been extensively used to evaluate the effects of temperature on cardiovascular disease (CVD). However, few studies have analyzed and compared their effects on CVD in less-developed, rural areas of China.

\section{Methods}

A case-only analysis was conducted of 43,567 cases of CVD morbidity in Qingyang (Northwest China) in 2011-2017 and the effects of thermal indicators were assessed using distributed lag nonlinear modelling and a Poisson regression model. Data on CVD morbidity originated from the New Rural Cooperative Medical Insurance of Gansu Province and meteorological variables were provided by the Meteorological Science Data Sharing Service.

\section{Results}

Both AT and DTR had significant nonlinear and delayed impacts on hospital admissions for CVD. DTR had a stronger and more persistent effect on CVD incidence than AT. Women were more affected by high AT and low DTR than were men, while men were more vulnerable to low AT and high DTR. Temperature effects were not significantly different between people above and below 65 years of age.

\section{Conclusions}

These findings provide local public health authorities with reference data concerning sensitive temperature indices for susceptible populations with a view to improving CVD preventive strategies in rural areas.

\section{Introduction}

Climate change is increasing the incidence of extreme weather events which have a significant impact on public health [1]. It was well-established that extreme weather events are one of the most important factors leading to increased morbidity and mortality [2,3]. The effects of temperature variations on cardiovascular disease (CVD), the leading cause of death [4-6] and the most preventable noncommunicable disease worldwide [7], have become a focus of research. Previous studies have reported that CVD mortality is highly sensitive to apparent temperature (AT) $[8,9]$, with nonlinear and reverse $\mathrm{J}$-shaped correlations being demonstrated $[10,11]$. Diurnal temperature range (DTR) is generally used to evaluate temperature impacts on CVD. For example, in China [12, 13], Korea [14] and USA [15] DTR was found to have nonlinear relationships with CVD morbidity and mortality. 
Most Chinese studies on the relationship between AT and CVD morbidity have been conducted in cities $[16,17]$, with few studies being performed in rural areas. Farmers are more susceptible to temperature changes compared with urban residents [18]. In rural Northwest China, Ding investigated associations between DTR and blood pressure [19]. Wang studied the impact of ambient temperature (rather than apparent temperature) on CVD in farmers [20]. These studies provided limited information on the differing impacts of DTR and AT on farmers in China. In less-affluent areas of Northwest China where medical facilities are relatively poor, it is necessary to identify appropriate weather indicators for early warning of cardiovascular disease-prone populations. The objective of this study was to assess and compare the efficacy of two temperature indicators of CVD morbidity among farmers in Qingyang between 2011 and 2017. This exploration of the relationship between thermometric variables and CVD risk may help local public health departments develop sensitive temperature indicators for susceptible populations and to instigate alleviation and prevention strategies [21].

\section{Materials And Methods}

\subsection{Location}

Qingyang is an eastern city of Gansu Province, Northwest China, at latitude $35^{\circ} 15^{\prime} \mathrm{N}$ and longitude $106^{\circ} 20^{\prime} \mathrm{E}$. It has $700 \mathrm{~km}^{2}$ of fertile fields and is known as "the granary of Gansu Province" as well as the city in China with the second largest energy resources. In 2019, the permanent population of Qingyang was 2.28 million, of which farmers accounted for approximately $60 \%$. It has a typically warm temperate continental monsoon climate with distinct alternating cold winters and warm summers (average annual temperatures $9.5-10.7^{\circ} \mathrm{C}$ ).

\subsection{Data collection}

Electronic medical records of outpatient admissions for CVD were reviewed at the New Rural Cooperative Medical Insurance of Gansu Province (NRCMI), the government agency responsible for farmer health data collection in Gansu Province. These records encompassed 30 Qingyang city and county hospitals covering 1st January 2011 to 27th March 2017 and included patients' sex, age, hospitalization time, residence, and disease classification. Diagnoses were coded according to the 10th edition of the International Classification of Diseases (ICD-10). CVD classifications included chronic rheumatic heart disease (105-109), hypertension (110-115), ischemic heart disease (I20-125) and other types of heart disease (130-152). It should be noted that due to a lack of age data, age group analyses were limited to 2014-2017. The study protocol was approved by the ethics committee of Gansu Provincial Hospital. The data is encrypted, because the patient's name, address, and contact information are all hidden and an informed consent waiver is obtained from the ethics committee. The study was performed in accordance with the Declaration of Helsinki.

Meteorological data were collated from the China Meteorological Science Data Sharing Service (http://data.cma.cn/). These included mean temperature ( $T_{\text {mean }}$ ), minimum and maximum temperatures 
( $T_{\max }$ and $\left.T_{\min }\right)$, wind speed (WS), relative humidity $(R H)$, and atmospheric pressure. Weather variables were measured at four fixed-site stations distributed evenly across Qingyang City. Daily mean concentration of air pollutants in the city from 2014 to 2017 was obtained from the China National Environmental Monitoring Center (http://webinterface.cnemc.cn/) and included PM2.5, PM10, SO2, NO2, $\mathrm{O} 3$ (average concentration for $8 \mathrm{~h}$ ), and CO (all units $\mu \mathrm{g} / \mathrm{m} 3$ ).

\subsection{Temperature indicators}

Several indicators of temperature changes, including DTR and AT, have proven effective. These two independent indicators were compared in this study. DTR was calculated by difference between daily minimum temperature and maximum temperature [22]. AT was calculated from common meteorological indicators using the following equations [23].

$$
\begin{aligned}
& A T=T+0.33 \times e-0.70 \times W S-4.00 \\
& e=R H / 100 \times 6.105 \times e^{[17.27 \times T /(237.7+T)]}
\end{aligned}
$$

Where $\mathrm{T}$ is daily mean temperature $\left({ }^{\circ} \mathrm{C}\right)$, WS is wind velocity $(\mathrm{m} / \mathrm{s})$, e is water vapor pressure $(\mathrm{hPa})$, and $\mathrm{RH}$ is relative humidity (\%)

\subsection{Statistical analysis}

Poisson generalized linear regression modelling combined with distributed lag nonlinear modelling (DLNM) was applied to examine the impact of temperature variables on CVD admissions. DLNM using the cross-basis function can simultaneously measure nonlinear exposure-response dependencies and delayed effects [24]. Cubic spline smoothing was adopted to explain variations in the temporal behavior of potential predictors. The smoothed predictors, that is the meteorological indicators, were added in a fashion similar to multiple linear regression. This approach is commonly applied in weather epidemiology and has been used in similar studies $[25,26]$. The general model $Y t \sim$ quasi-Poisson $(\mu \mathrm{t})$ was as follows:

$$
\begin{aligned}
\log \left(\mu_{t}\right)= & \alpha+\beta\left(\mathrm{X}_{t, l}\right)+n s\left(\text { Temperature }_{t}, d f\right)+n s(\text { Time }, d f)+\text { Holiday } \\
& +n s\left(L P_{t}, d f\right)+n s\left(W S_{t}, d f\right)+n s\left(R H_{t}, d f\right)+\text { Dow }
\end{aligned}
$$

where $Y t$ is the daily observed count of emergency visits to CVDs for day $t(t=1,2,3 \ldots 21)$, $a$ is the intercept, $X_{t, l}$ is the cross-basis matrix of temperature indicators in DLNM (AT and DTR were compared in two dependent models), $L$ is lag days, $\beta$ is the vector of the coefficients for temperature variations, $L P$ is local pressure, WS is wind speed, $\mathrm{RH}$ is relative humidity (meteorological factors were applied in the model using a natural cubic spline to control their potential implications), ns is natural cubic spline, and 
$\mathrm{df}$ is degrees of freedom, while the dummy variables DOW (day of the week) and Holiday were used to control the impact of the sampling day.

Previous studies proved that long-term tendency, local pressure, relative humidity, and wind speed were potential confounders having an impact on CVD outpatient visits [27-29]. Natural cubic splines were applied in DLNM to estimate the independent impact of temperature variations on outpatient admissions for CVD (three equally spaced knots were placed per year for the continuous dependent variables). A 3-df natural cubic spline was used to control the effects of humidity, wind speed, and local pressure [30,31], 4df to eliminate the impacts of mean temperature [32,33], and 7-df to remove long-time tendency [32,34]. Some studies have demonstrated that air pollutants are also associated with CVD [35]. However, in rural regions the impact of air pollution does not appear to be significant $[36,37]$ as concentrations of air pollutants are very low [38]. Furthermore, air pollutant sensitivity analysis was performed (Figure S1, see additional file 1) and no differences were observed. Air pollution was not included in the model in order to avoid overfitting. The degrees of freedom for DTR and lag in the cross-basis matrix were chosen according to a modified Akaike Information Criterion (Table S1, see additional file 2): 4-df for AT and 3-df for lag in AT cross-basis matrix and 6-df for DTR and 3-df for lag in DTR cross-basis matrix.

All data were stratified according to sex (male and female) and age (adult and old) for subgroup analyses. The threshold for age was set at 65 years-China's retirement age. The median value of the temperature indicators was used as the reference to calculate relative risk (RR) with a $95 \%$ confidence interval. Cumulative lag effect of the thermal indicators was assessed with a 21-day lag $[39,40]$. Various lag periods $(0,3,7,14$, and 30 days) were examined to determine the impact of temperature indicators on CVD visits. The impact of lagx denotes the effect of DTR on CVD incidence after $x$ days. Previous studies suggest that there is a cumulative lag effect of DTR on CVD admissions [31,41]. Most studies used a lag of 21 or $28 d[25,40,42]$. However, the current study assessed a lag of zero to 30 days to evaluate the cumulative effect of a month-long lag. Moreover, to assess the robustness of the model, several $\mathrm{df}$ of the meteorological variables were evaluated in a sensitivity analysis, including temperature $(\mathrm{df}=3-5)$, relative humidity $(d f=4-6)$, local pressure $(d f=4-6)$ and wind speed $(d f=3-5)$, (Fig S2, see additional file 3). All statistical analyses and chart preparation were performed using the 'dlnm' package in the R v.3.4.1 statistical software.

\section{Results}

\section{Table 1 Summary statistics for daily variables and cardiovascular diseases (CVD) cases in Qingyang, China, 2011-2017}

Descriptive statistics for CVD cases and meteorological variables are presented in Table 1. A total of 43,567 cases of CVD were included in the study from 2011 to 2017, of which approximately 54\% were male and $46 \%$ were female. The subgroup categorized by age from 2014 to 2017 encompassed $71 \%$ of all cases (due to the incomplete age data). Adult patients accounted for $41 \%$ of these cases and old patients accounted for $59 \%$. The mean apparent temperature was $8.13^{\circ} \mathrm{C}$ (with a large standard deviation 


\begin{tabular}{|c|c|c|c|c|c|c|c|c|}
\hline \multirow[t]{2}{*}{ Variable } & \multirow[t]{2}{*}{ Sum } & \multirow[t]{2}{*}{ Mean } & \multirow[t]{2}{*}{$\mathrm{SD}$} & \multicolumn{5}{|c|}{ Frequency distribution } \\
\hline & & & & Min & $P(25)$ & $P(50)$ & $P(75)$ & Max \\
\hline All & 43567 & 20.5 & 15.08 & 0 & 9 & 18 & 29 & 211 \\
\hline Man & 23483 & 11.05 & 9.27 & 0 & 4 & 8 & 16 & 97 \\
\hline Woman & 20083 & 9.45 & 6.87 & 0 & 4 & 8 & 13 & 114 \\
\hline$<65$ years & 16736 & 14.16 & 9.58 & 0 & 7 & 13 & 20 & 59 \\
\hline$\geq 65$ years & 14263 & 12.07 & 7.32 & 0 & 7 & 12 & 17 & 65 \\
\hline Mean temperature $\left({ }^{\circ} \mathrm{C}\right)$ & / & 10.15 & 10.14 & -14.7 & 1 & 11.3 & 19.2 & 30.6 \\
\hline Relative humidity(\%) & / & 65.33 & 16.99 & 14 & 53 & 66 & 79 & 100 \\
\hline Pressure(hpa) & I & 892.87 & 6.27 & 877.6 & 887.8 & 892.7 & 897.6 & 916.3 \\
\hline Wind $\operatorname{speed}(\mathrm{m} / \mathrm{s})$ & I & 1.84 & 0.72 & 0.3 & 1.4 & 1.7 & 2.2 & 5.9 \\
\hline Rainfall(mm) & I & 1.47 & 5.32 & 0 & 0 & 0 & 0 & 67.7 \\
\hline AT $\left({ }^{\circ} \mathrm{C}\right)$ & I & 8.13 & 12.08 & 19.75 & -2.89 & 8.95 & 18.99 & 32.41 \\
\hline $\operatorname{DTR}\left({ }^{\circ} \mathrm{C}\right)$ & / & 12.82 & 5.46 & 1 & 8.7 & 12.9 & 16.9 & 29.6 \\
\hline
\end{tabular}

$12.08^{\circ} \mathrm{C}$ ) ranging from $-19.75^{\circ} \mathrm{C}$ to $32.41^{\circ} \mathrm{C}$, being slightly lower than the mean temperature $\left(10.15^{\circ} \mathrm{C}\right)$. The average DTR was $12.82^{\circ} \mathrm{C}$ (with a moderate standard deviation of $5.46^{\circ} \mathrm{C}$ )

Fig. 1 shows the 21-day cumulative lag effect of AT and DTR on the RR of CVD admissions in all groups. Overall, the impact of DTR on CVD was greater than of AT for all subgroups. The relationship between the temperature variables and the RR of CVD resembles a bimodal function. Table 2 (see additional file 2) shows the RR of CVD for AT and DTR. The first peak was evident at AT $-4^{\circ} \mathrm{C}$ or DTR $6^{\circ} \mathrm{C}(\mathrm{AT} 1.083, \mathrm{DTR}$ 2.243) and the second at AT $20^{\circ} \mathrm{C}$ or DTR $19^{\circ} \mathrm{C}$ (AT 1.596, DTR 1.875). DTR posed more of a risk of CVD incidence to men than to women when DTR was at a high of $19^{\circ} \mathrm{C}$ (male 2.061 , female 1.662), while this was reversed at a low DTR of $6^{\circ} \mathrm{C}$ (male 2.167, female 2.491). Women suffered more risk than men when AT was ${ }^{3} 20^{\circ} \mathrm{C}$ (AT $20^{\circ} \mathrm{C}$ : male 1.125 , female 2.220$)$, while the opposite held when AT $£ 0^{\circ} \mathrm{C}\left(\mathrm{AT}-4^{\circ} \mathrm{C}\right.$ : male 1.348, female 0.859). In the age-specific study, the maximum CVD RR occurred at the lowest DTR (adult 2.327 , old 2.368) and then increased to its maximum when DTR was $19^{\circ} \mathrm{C}$ (adult 2.527, old 2.752). The impact of AT on RR of CVD was most significant at $0^{\circ} \mathrm{C}$ (adult 1.508, old 1.497) and there was no significant difference between the age groups.

The single lag effect of the temperature indicators on RR of CVD was examined by contour map (Fig. 2, reference $12^{\circ} \mathrm{C}$ ). Overall, the AT models demonstrated a $2 \%$ increase in RR when AT rose from $16^{\circ} \mathrm{C}$ to $24^{\circ} \mathrm{C}$, but this disappeared after about two weeks. A weaker and shorter cold effect was observed from $-10^{\circ} \mathrm{C}$ to $0^{\circ} \mathrm{C}$. The heat effect also lasted longer. However, the RR of CVD admission decreased gradually by $1-5 \%$ across the lag days when DTR was $6^{\circ} \mathrm{C}$, while a $3 \%$ increment was sustained for a month when 
DTR was $19^{\circ} \mathrm{C}$. There was also a protective effect at high DTR ${ }^{3} 25^{\circ} \mathrm{C}$. Overall, DTR exerted a stronger and longer single lag impact on RR of CVD incidence in all people compared with AT (AT 1.020 lasting for 15 days; DTR 1.05 lasting for 22 days).

The AT single lag effect was more significant and longer with women (1.03) than with men (1.01) at AT above $20^{\circ} \mathrm{C}$, but there was no effect at colder temperatures. The adverse effects of DTR for men were greater and longer than for women at both low and high DTR (men 1.04 for one month, women 1.04 for half a month). Overall, the effect of AT on men was gradually attenuated and disappeared after five days, while the effect of DTR was longer and stronger. However, the lag effect of AT was more obvious on women. Men suffered a longer and stronger RR of CVD when exposed to high DTR than did women, while women experienced a longer lag effect at high AT than men.

A comparison of adult vs. old CVD incidence showed that DTR exerted a stronger single lag effect on CVD for both adults and old people (AT 1.04, DTR 1.075). Both age groups exhibited the same pattern of impact of DTR on CVD (adult 1.075, old 1.075). However, adults experienced a greater risk in days of low AT compared with the elderly (adult 1.04, old 1.02). The protective impact of temperature occurred at the extremes: below $-10{ }^{\circ} \mathrm{C}$ and above $30{ }^{\circ} \mathrm{C}$ for AT, but only at high DTR. The contour map of the effect of AT on age group was unimodal, while DTR was bimodal.

\section{Discussion}

To our knowledge, this study is the first to compare and analyze the effects of DTR and AT on the onset of CVD in rural areas. Both AT and DTR had significant nonlinear, delayed effects on CVD morbidity. Whether cumulative or single lag, the effect of DTR on CVD incidence was greater and more persistent than AT in all groups. Women were more affected by AT above $20^{\circ} \mathrm{C}$ than men, but this was reversed at AT below $0^{\circ} \mathrm{C}$. However, men appeared to be more vulnerable to high DTR $\left(19^{\circ} \mathrm{C}\right)$ than women, while this was reversed at low DTR $\left(6^{\circ} \mathrm{C}\right)$. There was no difference in cumulative lag impact on age subgroups in both the DTR and AT models, but the single lag impact of AT on adult men was greater than on the elderly.

We found that both DTR and AT had significant impacts on cardiovascular morbidity, while the relationship between the thermal variables and risk of CVD was nonlinear with M-shaped curves. However, this was inconsistent with many prior studies that reported relationships between AT and RR of CVD having reverse J-shaped curves $[10,11,16]$. This was also the case for DTR. Youn-Hee demonstrated a positive linear relation between DTR and Emergency Room admissions in Korea [14, 34]. J-, V- and Ushaped correlations between DTR and CVD have been demonstrated in USA [15], China [12, 13], and Korea [14]. These differences may be due to geographical effects, climatic characteristics, differences in use of cooling and heating equipment, and housing types [13].

Overall, DTR was associated with a greater risk and disease burden of CVD (cumulative lag effect: DTR 2.37, AT 1.62). Few studies have compared the extent of the effects of AT and DTR. Increases of $33 \%$ and $3 \%$ in cumulative DTR-related risk of CVD were associated with $8.2^{\circ} \mathrm{C}$ and $1^{\circ} \mathrm{C}$ ranges in southwest China 
[41] and Korea [14], respectively. The risk of CVD mortality increased by $7 \%$ and $14 \%$ with $7{ }^{\circ} \mathrm{C}$ and $8^{\circ} \mathrm{C}$ increases in AT in Iran [10] and Copenhagen [11], respectively. A study conducted in Virginia, USA, showed that DTR was associated with a higher incidence of Emergency Department visits than were AT and mean temperature [42]. These studies support the current finding that the RR of CVD was stronger and longer under the influence of DTR than AT. The differences in the impact of AT and DTR on CVD can be attributed to their differing pathogenesis. DTR affects cardiovascular mortality through alterations in autonomic nervous functions. A large DTR can induce disturbances of the autonomic nervous system, resulting in elevated heart rate and decreased heart rate variability, which indicate impacts on the sympathetic and parasympathetic nerves and on sinoatrial node frequency (i.e., regulation of neurohumoral factors of the cardiovascular system)[14]. Furthermore, heart rate, cardiac workload and blood pressure changes triggered by wider temperature changes have been shown to contribute to the onset of CVD $[19,20]$. AT-related impacts on CVD can be explained by that body's circulatory system responding to high or low temperatures by releasing platelets into circulation and the increasing red and white cell counts, blood viscosity and plasma cholesterol resulting from water loss and reduced plasma volume under hot conditions [21]. The direct effects of DTR on the autonomic nervous system and the indirect effects of AT on the circulatory system explain the differing impact and lag periods of these temperature parameters.

Danet et al. demonstrated that a $1.3 \%$ increase in fatal coronary events was associated with a $1^{\circ} \mathrm{C}$ decrease in DTR in USA [42], while a $1^{\circ} \mathrm{C}$ increase in mean AT was associated with a $2.4 \%$ increase in cardiovascular events in Portugal [8]. These studies suggest that AT is associated with a higher risk of CVD than DTR, which is not consistent with the current study. Possible reasons for this inconsistency are differences in population demographics, exposure conditions, climatic characteristics, and climate-related behaviors [11, 31, 44]. The current study area, Qingyang located in the Loess Plateau, has a continental climate which creates a large DTR but a relatively moderate AT. People in Qingyang suffer a greater risk of CVD due to DTR rather than AT because they are exposed to large temperature differences rather than extreme temperatures. Furthermore, farmers, especially those most susceptible to cardiovascular disease, tend to be more vulnerable to DTR because of their lack of awareness of how to protect against extreme DTR. Moreover, the weather forecast broadcasts temperature rather than DTR. People suffer more adverse effects from DTR than AT because it can be hard to respond quickly to sudden temperature changes, while people can deal with high and low temperatures by adding or removing clothing in advance, using air conditioning, and so on. It is proposed that relevant departments should broadcast DTR forecasts for the benefit of CVD-susceptible populations.

Another interesting finding in this study was that women were susceptible to a slightly higher AT than men, whereas, when AT was below $10^{\circ} \mathrm{C}$ men were at higher risk. Previous studies are consistent this finding, confirming that women have a higher risk of CVD in hot conditions, while men are at greater risk in cold conditions $[10,15,27]$. This may be due to women being more susceptible to arrhythmia, ischemia, and high blood pressure, all of which are exacerbated by extreme hot and cold temperatures $[21,45]$. Conversely, hot conditions increased coronary events in men in San Paulo while low temperatures with up to two lag days increased women's mortality risk in a subtropical climate zone in 
China [45]. Meta-analysis performed by Moghadamnia et al. on data from Mohamamad et al. confirmed their finding of no difference in the impact of hot and cold temperatures on CVD in the two genders [28, 45]. Basu suggested that different gender temperature effects were the result of location and population [46]. A European study demonstrated that clothing plays a significant role in such differences and that there are also biological gender differences in thermoregulation [47]. Thus, women should pay more attention than men to protective measures when $A T>20^{\circ} \mathrm{C}$. A further issue is that farmers, whether women or men, have increased exposure to high AT during the harvest and sowing seasons in mid-April and mid-September [48-50] when temperatures range from 20 to $25^{\circ} \mathrm{C}$ (Fig S3, see additional file 4). Both men and women work during these periods but women bear the greater risk of CVD [51]. Again, clothing was shown to play a significant role in this gender difference [30].

DTR-related RR of CVD in men was higher than in women at high DTR $\left(20^{\circ} \mathrm{C}\right)$, while this was reversed at low DTR $\left(5^{\circ} \mathrm{C}\right)$. Epidemiological studies have proven that CVD mortality in females was more strongly associated with DTR than in males in China [13,52], Korea [10], and Japan [53]. This is in line with the present results at low DTR, but conflicts with the results at high DTR. This sex-related difference may be attributable to demography-related behavioral differences and geographical effect modifiers [10]. The present data were obtained from the NRCMI in order to examine a population of farmers. In rural Qingyang more than $30 \%$ of farmers are migrant workers [54-56] and most of them are men whose work is usually outdoors, while the women generally work at home. The woman could, therefore, be more flexible at work compared with the men in the face of adverse weather conditions, possibly decreasing or avoiding exposure during high-DTR days, while the men remained exposed to the adverse temperatures. Consequently, on suitable days with low DTR, women may go out to work along with the men but they also bear a greater CVD risk due to sex-related physical differences [51]. However, the men had longer exposure on the high-DTR and low-AT days, leading to a higher risk of CVD compared with women. A previous study has shown that greater DTR exposure or weaker intrinsic susceptibility factors may result in increased risk [14]. Different subgroups and mortality categories were sensitive to different temperature indicators [57], which also illustrates why women were sensitive to high AT and men were sensitive to high DTR.

To our knowledge, this is the first study to compare and analyze the impact of DTR and AT on CVD. The relationship between thermal variations and morbidity was studied in a developing rural area of Northwest China (Qingyang). This study used validated and credible data originating from NRCMI of Gansu Province which recorded all outpatient visits by farmers. The results provide information for local government to enhance the protection of citizens of rural and less-developed areas. This analysis has both strengths and limitations. Firstly, only one area was selected as representative of typically poor rural regions. Further studies need to be conducted to evaluate DTR impact in other rural regions and confirm these findings. Secondly, the effects of characteristics such as personal behavior, medical history and living conditions were not considered due to their absence from the available data. Thirdly, the assumption that all individuals were exposed to the same thermal environment was a generalization. Finally, the retrospective data collection method may allow biases from diagnostic and coding inaccuracies. 


\section{Conclusion}

This study investigated and compared the effects of DTR and AT on the incidence of CVD in a rural and less-developed area of Northwest China using DLNM. The effect of DTR was larger and more persistent than AT. Women were more sensitive (and for longer periods) to high AT and low DTR compared with men, while men were more susceptible to low AT and high DTR. However, there was no significant difference in the effects of the two temperature indices on CVD in different age groups. Since temperature-associated adverse effects are potentially modifiable via preventive measures and lifestyle changes, these findings may have important implications for the prevention of cardiovascular disease in less-developed rural areas.

\section{Abbreviations}

DTR: Diurnal temperature range; AT: Apparent temperature;

CVD: Cardiovascular diseases;

NRCMI: New Rural Cooperative Medical Insurance of Gansu Province;

DLNM: distributed lag nonlinear model. RR: relative risk of CVD.

\section{Declarations}

\section{Availability of data and material}

The datasets used and/or analysis during the current study available from the corresponding author on reasonable request.

\section{Acknowledgements}

This was supported by China Postdoctoral Science Foundation (No.2016M600827) ,the National Natural Science Foundation of China (No.71861026), Gansu Science and Technology Program 'Knowledge-driven Meteorological Environment for Public Health Economic Loss Evaluation' (No.16JZD023) and Gansu Natural Science Foundation Project ' Evaluation and Spatial Differentiation of Green Competitiveness in Gansu Province from the Perspective of Ecological Civilization ' (No.20JR5RA474). The authors would like to express their gratitude to Edit Springs for the expert linguistic services.

\section{Authors' contributions}

Zhai contributed to design the conception of the study and analysis and interpret the data. Qi contributed to the acquisition of data and drafting the article, and the important intellectual content was revised by Zhang. Zhou and Wang participated in the final approval of the version to be submitted. All authors have approved the manuscript. 


\section{Ethics approval and consent to participate}

The study protocol was approved by the ethics committee of Lanzhou University of Technology. The data is encrypted, the patient's name, address, and contact information are all hidden and informed consent waiver obtained from the ethics committee. The study was performed in accordance with the Declaration of Henlsinki.

\section{Consent for publication}

All consent.

\section{Competing interests}

The authors declare that they have no competing interests.

\section{Authors' information}

Not applicable.

\section{Funding}

China Postdoctoral Science Foundation (No.2016M600827) ,the National Natural Science Foundation of China (No.71861026), Gansu Science and Technology Program 'Knowledge-driven Meteorological Environment for Public Health Economic Loss Evaluation' (No.16JZD023) and Gansu Natural Science Foundation Project ' Evaluation and Spatial Differentiation of Green Competitiveness in Gansu Province from the Perspective of Ecological Civilization ' (No.20JR5RA474).

\section{References}

1 Rumsey M, Fletcher SM, Thiessen J, et al. A qualitative examination of the health workforce needs during climate change disaster response in Pacific Island countries. Hum Resour Health. 2014;12:9.

2 Ghiasmand M, Moghadamnia MT, Pourshaikhian M, Lili EK. Acute triggers of myocardial infarction: a case-crossover study. Egypt Heart J. 2017;69:223-228.

3 Wilson LA, Morgan GG, Hanigan IC, et al. The impact of heat on mortality and morbidity in the greater metropolitan Sydney region: a case crossover analysis. Environ Health. 2013;12:98.

4 Michelozzi Paola, Accetta Gabriele, De Sario Manuela et al. High Temperature and Hospitalizations for Cardiovascular and Respiratory Causes in 12 European Cities. AM J RESP CRIT CARE.2009;179:383-389.

5 MENKES MS, MATTHEWS KA, KRANTZ DS et al. Cardiovascular Reactivity to the Cold Pressor Test as a Predictor of Hypertension. Hypertension.1989;14:524-530. 
6 Lin Shao, Luo Ming, Walker Randi J et al. Extreme High Temperatures and Hospital Admissions for Respiratory and Cardiovascular Diseases. Epidemiology.2009;20:738-746.

7 Ghiasmand M, Moghadamnia MT, Pourshaikhian M, Lili EK. Acute triggers of myocardial infarction: a case-crossover study. Egypt Heart J. 2017;69:223-228.

8 Sofia P Almeida, Elsa Casimiro, José Calheiros. Effects of apparent temperature on daily mortality in Lisbon and Oporto, Portugal. Environmental Health.2010;9:12.

9 Goran Krstic. Apparent Temperature and Air Pollution vs. Elderly Population Mortality in Metro Vancouver Environmental Health Services.2011;6:e25101.

10 Mohammad Taghi Moghadamnia, Ali Ardalan, Alireza Mesdaghinia et al: The Effects of Apparent Temperature on Cardiovascular Mortality Using a Distributed Lag Nonlinear Model Analysis: 2005 to 2014. Asia Pacific Journal of Public Health.2018;30:361-368.

11 Wichmann J, Andersen ZJ, Ketzel M, Ellermann T, Loft S et al: Apparent Temperature and CauseSpecific Mortality in Copenhagen, Denmark: A Case-Crossover Analysis. Int J Env Res Pub He. 2011;8:3712-3727.

12 Kan H, London SJ, Chen H, Song G, Chen G, Jiang L, Zhao N, ZhangY, Chen B (2007) Diurnal temperature range and daily mortality in Shanghai, China. Environ Res 103:424-431.

13 Xiaodan Zhou, Ang Zhao, Xia Meng, Renjie Chen.et al. Acute effects of diurnal temperature range on mortality in 8 Chinese cities. Sci Total Environ 2014;493:92-97.

14 Youn-Hee, Lim, Yun-Chul ,Hong, Ho, Kim: Effects of diurnal temperature range on cardiovascular and respiratory hospital admissions in Korea. Sci Total Environ 2012, 417:55-60.

15 K.L.Ebi·K.A.Exuzides·E.Lau·M.Kelsh·A. Barnaton: Weather changes associated with hospital-lizations for cardiovascular diseases and stroke in California, 1983-1998. Int J Biometeorol 2004, 49:48-58.

16 Liu Junhan, Li Geying, Mo Yunzheng. Relationship between Ambient Apparent Temperature and Daily Mortality of Population in a District of Beijing. Journal of Environment and Health.2011;12:1044-1047.

17 Mo Yunzheng, Xu Meimei, Jin Xiaobin, Pan Xiaochuan, Ma Wenjun. Association between Ambient Apparent Temperature and Cause-specific Mortality of Ischemic Heart Diseases in Beijing,China. Journal of Environment and Health.2012;5:387-391.

18 Mutekwa, V. Climate change impacts and adaptation in the agricultural sector: the case of smallholder farmers in Zimbabwe. Journal of Sustainable Development in Africa. 2009;11:237-256.

19 Shan Zheng, Wenzhi Zhu, Minzhen Wang, Qin Shi,et al. The effect of diurnal temperature range on blood pressure among 46,609 people in Northwestern China. Sci Total Environ. 2020;730:138987. 
20 Wang, B, Chai, GR and Sha, YZ et nl: Impact of ambient temperature on cardiovascular disease hospital admissions in farmers in China's Western suburbs. Sci Total Environ 2020, 761:263-254.

21 Yang J, Yin P, Zhou M, et al. Cardiovascular mortality risk attributable to ambient temperature in China. Heart. 2015;101:1966-1972.

22 Xu, Z., Huang, C., Su, H., Turner, L.R., Qiao, Z., Tong, S., 2013. Diurnal temperature range and childhood asthma: a time-series study. Environ. Health 12, 12.

23 Krstić, G., 2011. Apparent temperature and air pollution vs. elderly population mortality in Metro Vancouver. PLoS One 6 (9), e25101.

24 Gasparrini, A., 2014. Modeling exposure-lag-response associations with distributed lag non-linear models. Stat. Med. 33 (5), 881-899.

25 Guo, Y., Gasparrini, A., Armstrong, B., Li, S., Tawatsupa, B., Tobias, A., 2016. Temperature variability and mortality: a multi-country study. Environ. Health Perspect. 124 (10), 1554-1559.

26 Lee, W., Bell, M.L., Gasparrini, A., Armstrong, B.G., Sera, F., Hwang, S., 2018. Mortality burden of diurnal temperature range and its temporal changes: a multi-country study. Environ. Int. 110, 123-130.

27. Zhao Q, Zhao Y, Li SS, Zhang YJ, Wang QG. et al: Impact of ambient temperature on clinical visits for cardio-respiratory diseases in rural villages in Northwest China. Sci Total Environ 2018, 612:379-385.

28. Wang QG, Zhao Q, Wang GQ. et al: The association between ambient temperature and clinical visits for inflammation-related diseases in rural areas in China. Environ Pollut 2020, 261:261.

29. Goggins WB, Chan EYY. A study of the short-term associations between hospital admissions and mortality from heart failure and meteorological variables in Hong Kong Weather and heart failure in Hong Kong. Int J Cardiol.2017;228:537-542.

30. Guo Y, Barnett, A.G, Pan X, Yu W, Tong S. The impact of temperature on morta-lity in Tianjin, China: a case-crossover design with a distributed lag nonlinear model. Environ. Health Perspect. 2011;119:1719e1725.

31. Song XP, Wang SG, Li TS. et al: The impact of heat waves and cold spells on respiratory emergency department visits in Beijing,China. Sci Total Environ. 2017; 615:1499-1505.

32. Jian Cheng, Zhiwei Xu, Hilary Bambrick, et al. The mortality burden of hourly temperature variability in fifive capital cities,Australia: Time-series and meta regression analysis. Environ Int. 2017;109:10-1

33. Qi Zhao, Yi Zhao, Shanshan Li, et al. Impact of ambient temperature on clinical visits for cardiorespiratory diseases in rural villages in northwest China. Sci Total Environ. 2018;612:379-385. 
34. Lim, Youn-Hee, Park, Ae Kyung, Kim, Ho. Modifiers of diurnal temperature range and mortality association in six Korean cities. Int J Biometeorol. 2012;56:33-42.

35. Hoek, G, Krishnan, R.M, Beelen, R, Peters, A, Ostro, B, Brunekreef, B,Kaufman, J.D. Long-term air pollution exposure and cardio-respiratory mortality: a review. Environ. Health. 2013;12:43.

36. Zan Ding, Pi Guo, Fang Xie, Huifang Chu, Kun Li, Jingbo Pu. Impact of diurnal temperature range on mortality in a high plateau area in southwest China: A time series analysis. Sci Total Environ. 2015;526:358-365.

37. Wen-Miin, Liang·Wen-Pin, Liu·Sze-Yuan, Chou·Hsien-Wen Kuo. Ambient temperature and emergency room admissions for acute coronary syndrome in Taiwan. Int J Biometeorol. 2008;52:223-229.

38. K.L.Ebi·K.A.,Exuzides·E.Lau·M.Kelsh·A. Barnaton. Weather changes associated with hospitalizations for cardiovascular diseases and stroke in California, 1983-1998. Int J Biometeorol. 2004;49:48-58.

39. Guo, Y, Gasparrini, A, Armstrong, B.G., Tawatsupa, B., Tobias, A., Lavigne, E., et al. Temperature variability and mortality: a multi-country study. Environ. Health Perspect. 2016;124:1554-1559.

40. Gasparrini, A., Guo, Y., Hashizume, M., Lavigne, E., Zanobetti, A., Schwartz, J., et al. Mortality risk attributable to high and low ambient temperature: a multi country observational study. Lancet 2015;386:369-375.

41. Ye XF, Wolff R, Yu WW.et al. Ambient temperature and morbidity: a review of epidemiological evidence. Environ Health Perspect. 2012;120:19-28.

42. Robert E. Davis, Erin S. Markle, Sara Windoloski, et al. A comparison of the effect of weather and climate on emergency department visitation in Roanoke and Charlottesville, Virginia. Environ Res. 191 (2020) 110065.

42. Jian Cheng, Zhiwei Xu, Hilary Bambrick, et al. The mortality burden of hourly temperature variability in fifive capital cities,Australia: Time-series and meta regr-ession analysis. Environ Int. 2017;109:10-1

41. Zan Ding, Pi Guo, Fang Xie, Huifang Chu, Kun Li, Jingbo Pu: Impact of diurnal temperature range on mortality in a high plateau area in southwest China: A time series analysis. Sci Total Environ 2015, 526:358-365.

42. Danet S, Richard F, Montaye M, Beauchant S, Lemaire B, Graux C,et al (1999) Unhealthy effects of atmospheric temperature and pressure on the occurrence of myocardial infarction and coronary deaths. $A$ 10-year survey: the Lille-World Health Organization MONICA Project (Monitoring Trends and Determinants in Cardio-vascular Disease). Circulation 100:e1-e7.

43. K. L. Ebi · K. A. Exuzides - E: Weather changes associated with hospitalizations for cardiovascular diseases and stroke in California. Int J Biometeorol (2004) 49:48-58. 
44. Zan Ding, Liujiu Li, Lanyan Xin et al: High diurnal temperature range and mortality: Effect modifification by individual characteristics and mortality causes in a case-only analysis. Sci Total Environ 2016, 544:627-634.

45. Jixia Huang, Jinfeng Wang, Weiwei Yu. The Lag Effects and Vulnerabilities of Temperature Effects on

Cardiovascular Disease Mortality in a Subtropical Climate Zone in China. Int. J. Environ. Res. Public Health 2014, 11, 3982-3994.

46. Basu R, Dominici F, Samet JM. Temperature and mortality among the elderly in the United States: a comparison of epidemiologic methods. Epidemiology. 2005; 16: 58-66.

47. Keatinge, WR, Donaldson, GC, Bucher, K. Cold exposure and winter mortality from ischaemic heart disease, cerebrovascular disease, respiratory disease, and all causes in warm and cold regions of Europe. Lancet 1997;349:1341-1346.

48. Dianyuan Ding: Evaluation and Optimization of Winter Wheat Cultivation Measures in Loess Plateau Based on RZWQM2 Simulation. PhD thesis. Northwest a\&f university; 2016.

49. Tao Zhan: Response and Simulation of Spring Maize Productivity to Meteorological Factors in Semiarid Area of Northwest China. PhD thesis. Gansu agricultural university; 2018.

50. Yiwen Jiang: Applicability evaluation of DSSAT model in Heihe River Basin and application research of water-saving irrigation.PhD thesis. lanzhou university; 2015.

51. Hong, Y.C.; Rha, J.H.; Lee, J.T.; Ha, E.H.; Kim, H. Ischemic stroke associated with decrease in temperature. Epidemiology 2003, 14, 473-478.

52. Breitner S, Wolf K, Devlin RB, Diaz-Sanchez D, Peters A, Schneider A. Short-term effects of air temperature on mortality and effect modification by air pollution in three cities of Bavaria, Germany: a time-series analysis. Sci Total Environ. 2014;485-486:49-61.

53. Jayeun Kim, Jihye Shin, Youn-Hee Lim, Yasushi Honda, et al. Comprehensive approach to understand the association between diurnal temperature range and mor-tality in East Asia. Sci Total Environ. 2016; 539:313-321.

54. Tingjian,Guo and Jianmin, Han: Discussion on the transfer of rural surplus labor force in Qingyang city. Gansu Ag r. Sci. and Techn. No. 12011.

55. Zhiqiang, Wang: Investigation on education and training and employment status of migrant workers in Qingyang City. China Academic Journal Eletronic Publishing House.2011. 
56. Chunxiao, Hu: Study on the problem of migrant workers returning to their hometowns and returning to their hometowns under the vision of new countryside. China Academic Journal Eletronic Publishing House.2014.

57. Yu, Weiwei; Guo, Yuming; Ye, Xiaofang et al. The effect of various temperature indicators on different mortality categories in a subtropical city of Brisbane, Australia. Sci Total Environ 2011, 409(18): 34313437.

\section{Figures}
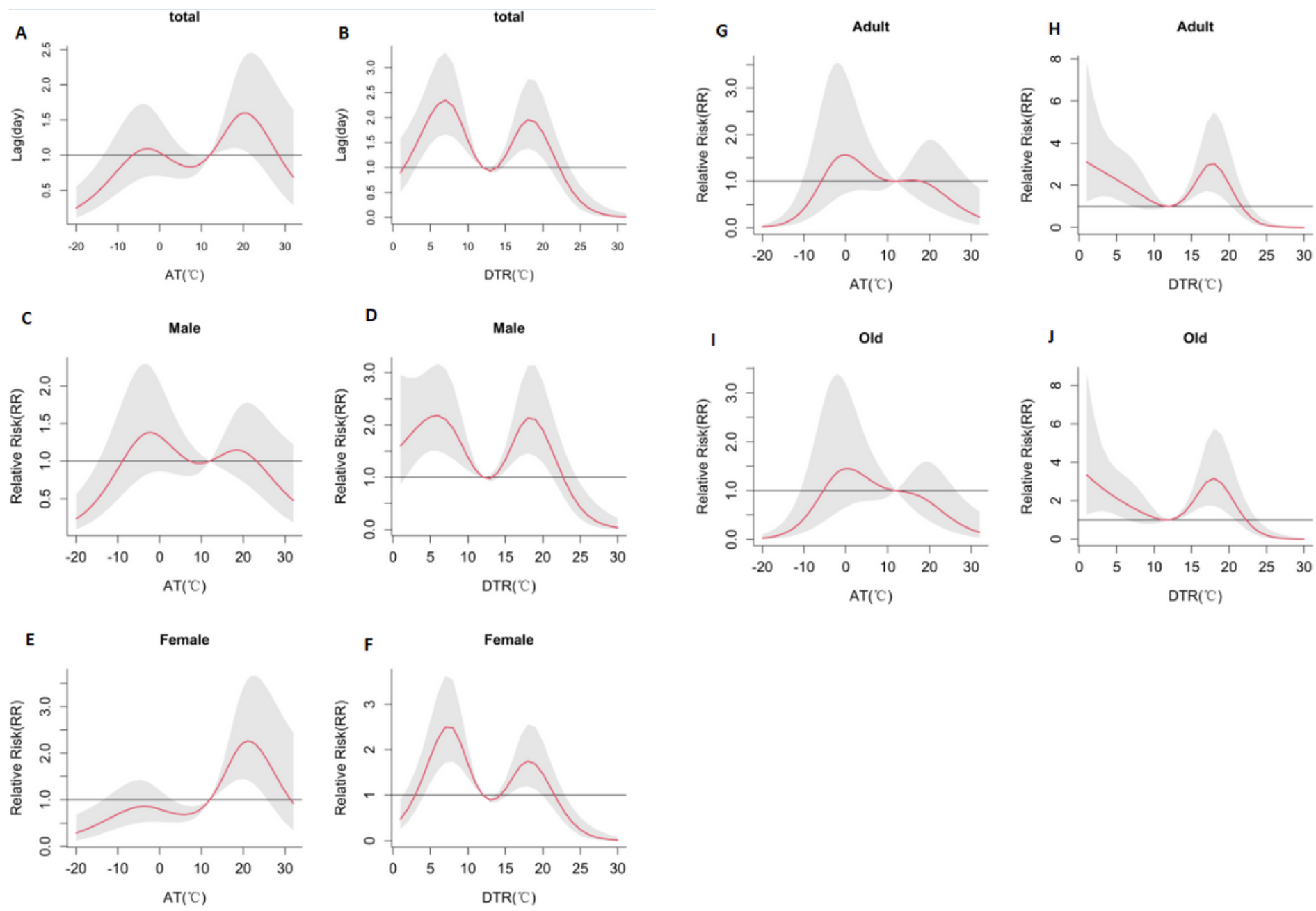

\section{Figure 1}

The 21-day cumulative lag effect of AT and DTR on the RR of CVD admissions in all groups 

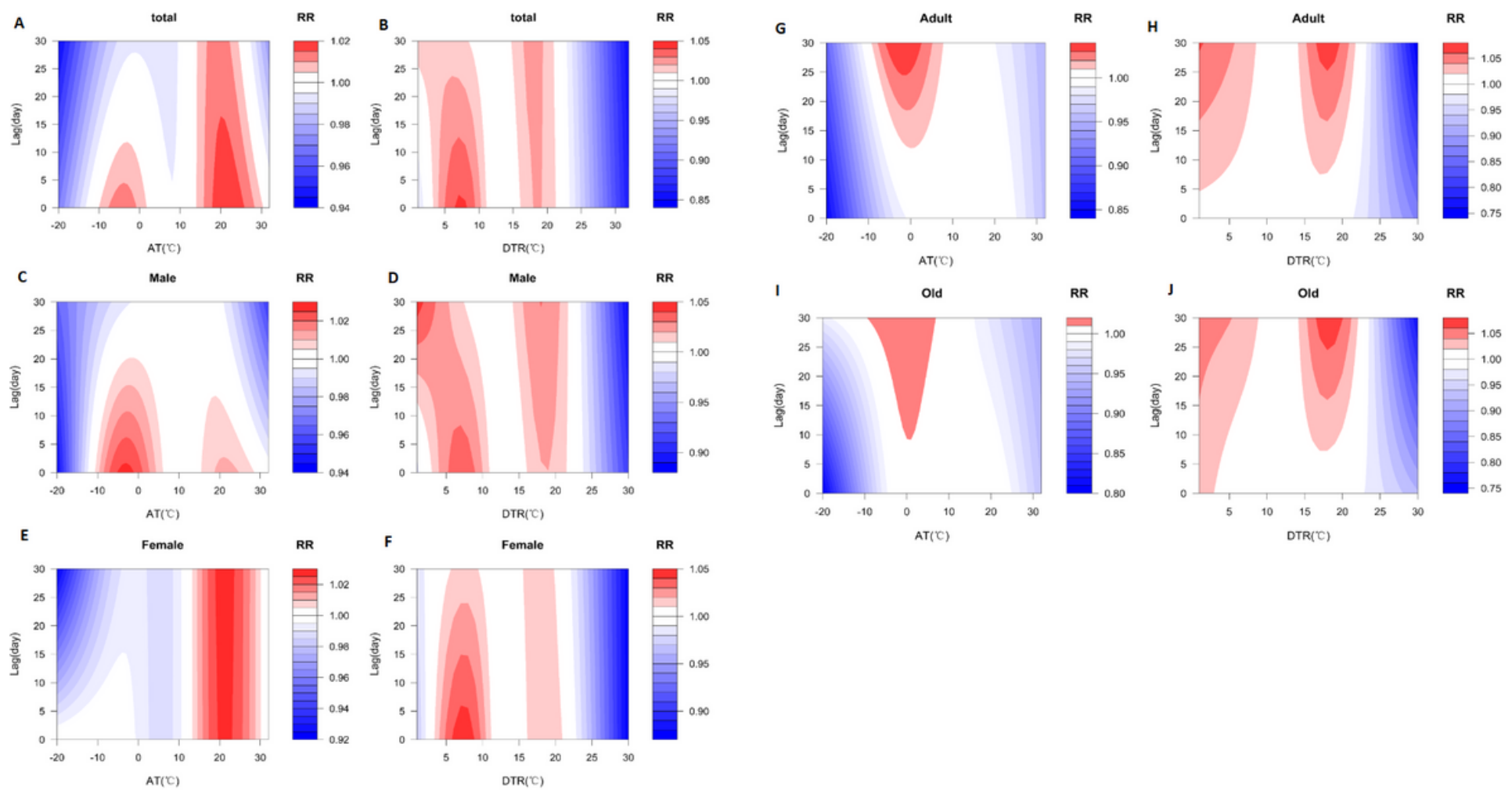

Figure 2

The contour map of single lag effect of the temperature indicators on RR of CVD

\section{Supplementary Files}

This is a list of supplementary files associated with this preprint. Click to download.

- Additionalfile1.tiff

- Additionalfile2.docx

- Additionalfile3.7z

- Additionalfile4.tiff 